\title{
Correlation and Path Coefficient Analysis of Cassava (Manihot esculenta Crantz) Genotypes
}

\author{
B. Babu Rao ${ }^{1 *}$, D.V. Swami ${ }^{1}$, P. Ashok ${ }^{1}$, B. Kalyana Babu ${ }^{3}$, \\ D. Ramajayam ${ }^{4}$ and K. Sasikala ${ }^{2}$ \\ ${ }^{1}$ Department of Horticulture, ${ }^{2}$ Department of Agronomy, Dr. Y.S.R. Horticultural University, \\ Venkataramannagudem, West Godavari, A.P - 534101, India \\ ${ }^{3}$ ICAR-Indian Institute of Oil Palm Research, Pedavegi, A.P - 534450, India \\ ${ }^{4}$ ICAR-National Research Centre for Banana, Tiruchirapally, T.N - 620102, India \\ *Corresponding author
}

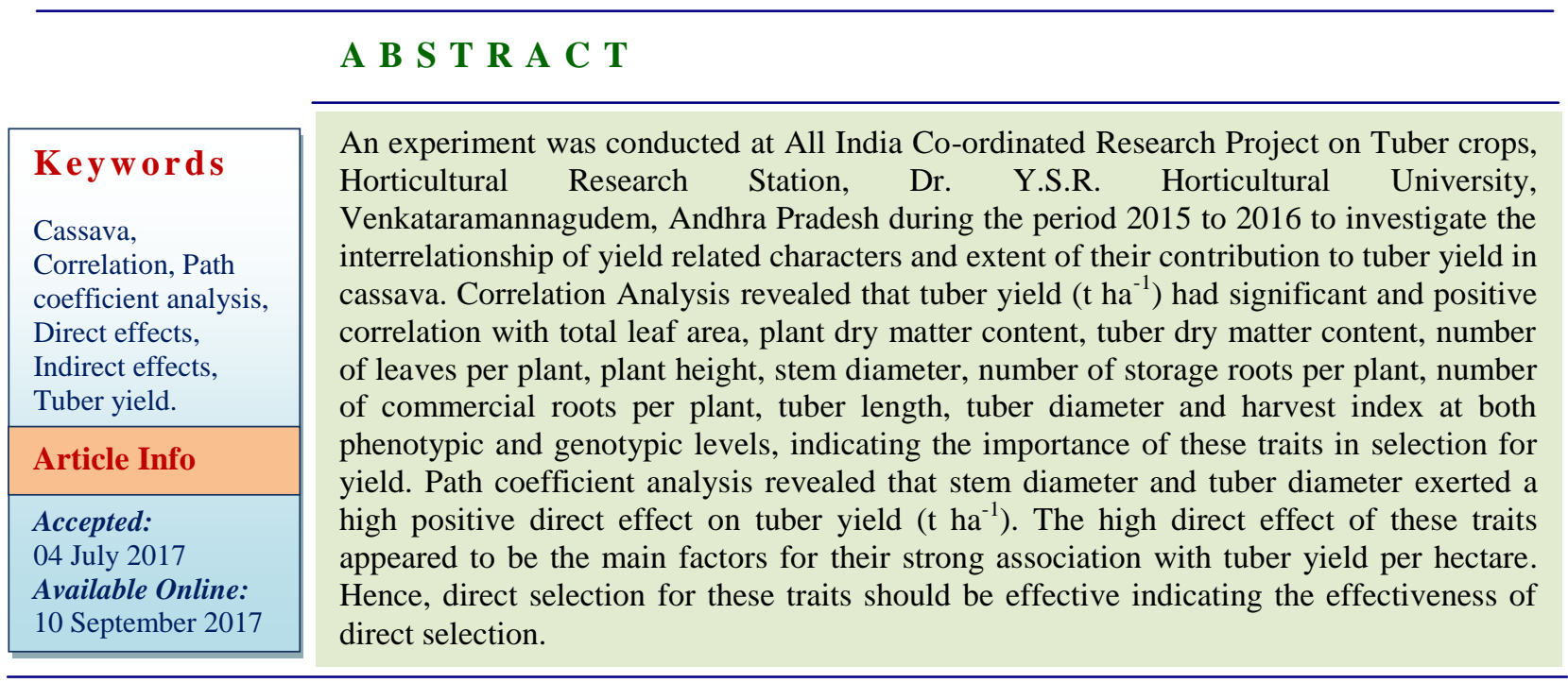

\section{Introduction}

Cassava (Manihot esculenta Crantz) is a perennial shrub, commonly known as tapioca, mandioca, manioc and yuca in different parts of the world. It belongs to the family Euphorbiaceae, sub family Crotonoidea and tribe Manihotae (Alves, 2002). The genus Manihot reported to have about 100 species of which Manihot esculenta is the only commercially cultivated species. It is native to North - Eastern Brazil. It is mainly cultivated for its starchy tubers and grown as staple food and animal feed in tropical and sub-tropical
Africa, Asia and Latin America. In Asia major pockets of cassava production at present are Indonesia, Thailand, India, China, Philippines and Vietnam. The crop was introduced into India from Brazil by the Portuguese when they landed in the Malabar region, presently part of Kerala state during the $17^{\text {th }}$ century. India acquires significance in the global cassava scenario due to its highest productivity in the world $\left(35.7 \mathrm{t} \mathrm{ha}^{-1}\right)$. It is being cultivated in an area of 2.29 lakh hectares in India, with a production of 81.4 
lakh MT and productivity of $35.7 \mathrm{t} \mathrm{ha}^{-1}$ (Anonymous, 2014). In Andhra Pradesh, it is being cultivated in East Godavari, Srikakulam, Vishakhapatnam and Vizianagaram districts with an area of 0.25 lakh hectares with a production of 4.42 lakh MT and productivity of $20.0 \mathrm{t} \mathrm{ha}^{-1}$ (Anonymous, 2014). Considering the economic importance of cassava and the low average productivity in Andhra Pradesh (20.0 $\mathrm{MT} / \mathrm{ha}$ ), there is tremendous scope to increase the yield per unit area and there by the total production.

Yield is a complex quantitative character controlled by several genes and its improvement depends largely on the functioning and interaction of several physiological components that vary for different genotypes. Apart from the direct selection for tuber yield, the purpose of root yield enhancement may, in most situations, be effectively achieved on the basis of performance of root yield components and selection for closely related morphophysiological characters (Kawano and Fakuda, 1998). There is a need to understand the inter character relationships among genotypes, identify traits that determine tuber yield and determine the influence of other traits associated with yield. Correlation analysis provides information about the degree of relationship between important plant traits and is a good index to predict yield response in relation to the change of a particular character. Genotypic and phenotypic correlations are of value to indicate the degree to which various morphophysiological characters are associated with productivity. Path coefficient analysis is a reliable statistical technique, providing a means to quantify the interrelationships of yield and yield-related as well as some other pathways to produce an effect (Kang, 1994). The present investigation was planned to examine associations among traits and identify characters correlated with tuber yield of cassava.

\section{Materials and Methods}

The experiment was conducted with seventy seven cassava genotypes along with three checks (Table 1) during the period from 2015 to 2016 at All India Co-ordinated Research Project on Tuber crops, Horticultural Research Station, Venkataramannagudem, Dr. Y.S.R. Horticultural University, Andhra Pradesh India. The location falls under the Agro-climatic zone number 10, East Coast Plain and Hills (Krishna-Godavari zone) at an altitude of $34 \mathrm{~m}$ (112 feet) above mean sea level with its geographical position $16.83^{\circ} \mathrm{N}$ latitude and $81.5^{\circ} \mathrm{E}$ longitude.

The experiment was laid out in Augmented Block Design (ABD) consisting of seven augmented blocks in which three checks and eleven entries were planted. Observations were recorded on five randomly selected plants for following traits i.e. petiole length, total leaf area, plant dry matter content, tuber dry matter content, number of leaves per plant, plant height, stem diameter, number of storage roots per plant, number of commercial roots per plant, tuber length, tuber diameter, harvest index, starch content, $\mathrm{HCN}$ content, post-harvest physiological deterioration and tuber yield.

\section{Statistical analysis}

Phenotypic and genotypic correlation coefficients between variables were calculated using covariance (Al-Jibouri et al., 1958 and Goulden, 1959). The significance of correlation coefficients was tested by comparing phenotypic correlation coefficients with table values (Fisher and Yates, 1963) at $n-2$ degrees of freedom. Direct and indirect contributions of various characters to tuber yield were calculated through path coefficient 
analysis according to Wright (1921) and elaborated by Dewey and Lu (1959).

\section{Results and Discussion}

The phenotypic $\left(\mathrm{r}_{\mathrm{p}}\right)$ and genotypic correlation $\left(r_{g}\right)$ coefficients for sixteen characters in 80 cassava genotypes were presented in table 2 and figure 1. In general, genotypic correlation coefficients were of higher magnitude than the phenotypic correlation coefficients and it is desirable also. This may be due to the relative stability of genotypes as majority of them were subjected to certain amount of selection (Johnson et al., 1955). In the present study tuber yield exhibited significant positive correlation with plant height $\left(0.269 \mathrm{r}_{\mathrm{p}}, 0.268\right.$ $\left.r_{g}\right)$, stem diameter $\left(0.395 r_{p}, 0.411 r_{g}\right)$, number of commercial tubers per plant $\left(0.322 \mathrm{r}_{\mathrm{p}}\right.$, $\left.0.327 \mathrm{r}_{\mathrm{g}}\right)$, tuber length $\left(0.420 \mathrm{r}_{\mathrm{p}}, 0.423 \mathrm{r}_{\mathrm{g}}\right)$, tuber diameter $\left(0.623 \mathrm{r}_{\mathrm{p}}, 0.641 \mathrm{r}_{\mathrm{g}}\right)$ and harvest index $\left(0.300 \mathrm{r}_{\mathrm{p}}, 0.315 \mathrm{r}_{\mathrm{g}}\right)$ at both 5 and $1 \%$ levels of significance. This character also recorded positive correlation with plant dry matter content $\left(0.208 \mathrm{r}_{\mathrm{p}}, 0.214 \mathrm{r}_{\mathrm{g}}\right)$, tuber dry matter content $\left(0.198 \mathrm{r}_{\mathrm{p}}, 0.204 \mathrm{r}_{\mathrm{g}}\right)$, total leaf area $\left(0.211 r_{p}, 0.209 r_{g}\right)$, number of leaves per plant $\left(0.207 r_{p}, 0.206 r_{g}\right)$ and number of roots per plant $\left(0.234 r_{p}, 0.238 r_{g}\right)$ at $5 \%$ level of significance only. These results were in conformity with research findings of earlier workers with respect to positive correlation of tuber yield with stem diameter $\left(0.395 \mathrm{r}_{\mathrm{p}}\right.$, $0.411 \mathrm{r}_{\mathrm{g}}$ ), number of commercial tubers per plant $\left(0.322 \mathrm{r}_{\mathrm{p}}, 0.327 \mathrm{r}_{\mathrm{g}}\right)$, tuber length $(0.420$ $r_{p}, 0.423 r_{g}$, and tuber diameter of $0.623 r_{p}$, $0.641 r_{\mathrm{g}}$ (Sree Kumari and Abraham., 1991; Nageswari and palaniswamy, 2011; Kundy et al., 2015; Babu Rao et al., 2015 and Danquah et al., 2016). Similar observations with regard to positive correlation of tuber yield with stem diameter were reported by Mulualem and Ayenew (2012). The correlation study indicated that the total leaf area, plant dry matter content, tuber dry matter content, number of leaves per plant, plant height, stem diameter, number of storage roots per plant, number of commercial roots per plant, tuber length, tuber diameter and harvest index had significant positive association with tuber yield at both phenotypic and genotypic levels. Hence, improvement in tuber yield is possible by considering above characters as criteria in selection scheme.

Fig.1 Simple correlation coefficients among yield and its components in cassava

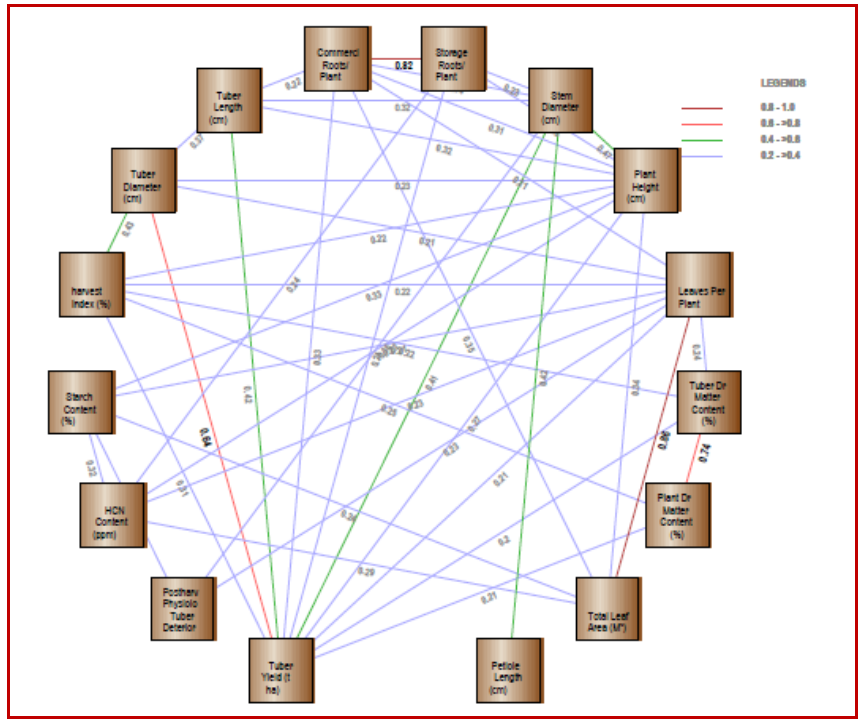


Fig.2 Genotypic path diagram representing direct and Indirect effects for tuber yield $\left(\mathrm{t} \mathrm{ha}^{-1}\right)$ of cassava

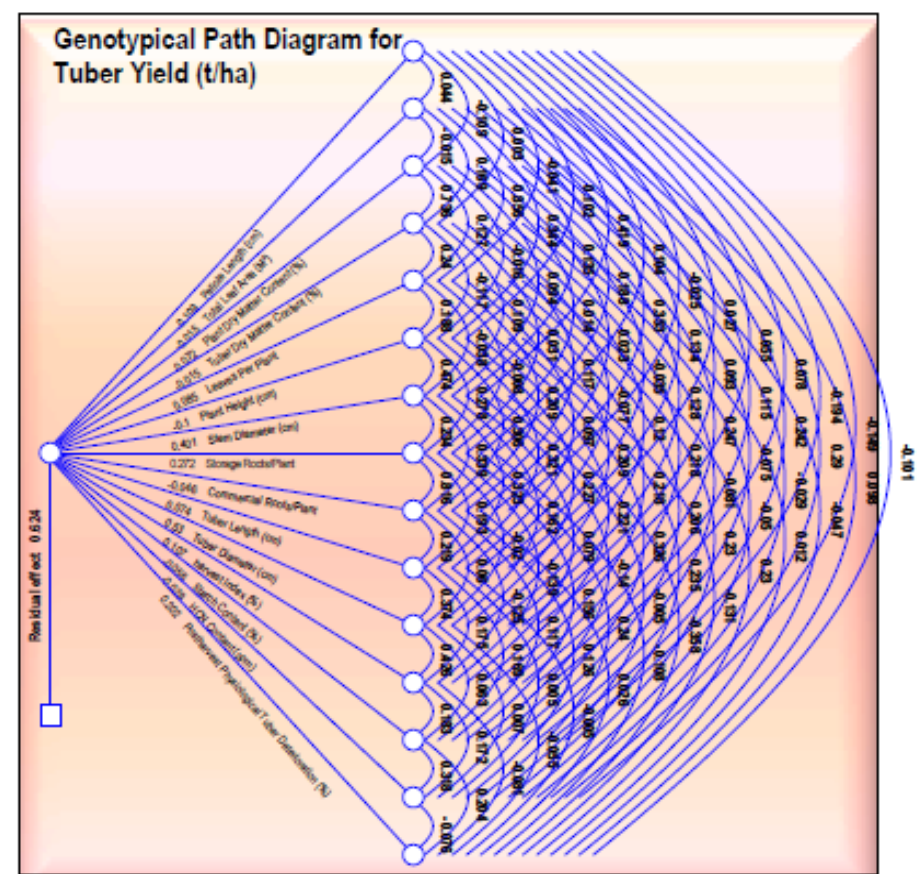

Table.1 Particulars of cassava genotypes under present study

\begin{tabular}{|c|c|c|}
\hline S. No. & Name of the genotype & Source \\
\hline 1. & Me Ap-1 & $\begin{array}{c}\text { AICRP on Tuber crops, Venkataramannagudem, Andhra } \\
\text { Pradesh }\end{array}$ \\
\hline 2. & Me Ap-2 & -do- \\
\hline 3. & Me Ap-3 & -do- \\
\hline 4. & Me Ap-4 & -do- \\
\hline 5. & Me Ap-5 & -do- \\
\hline 6. & Me Ap-6 & -do- \\
\hline 7. & Me Ap-7 & -do- \\
\hline 8. & Me Ap-8 & -do- \\
\hline 9. & Me Ap-9 & -do- \\
\hline 10. & Me Ap-10 & -do- \\
\hline 11. & Me Ap-11 & -do- \\
\hline 12. & Me Ap-12 & -do- \\
\hline 13. & Me Ap-13 & -do- \\
\hline 14. & Me Ap-14 & -do- \\
\hline 15. & Me Ap-15 & -do- \\
\hline 16. & Me Ap-16 & -do- \\
\hline 17. & Me Ap-17 & -do- \\
\hline 18. & Me Ap-18 & -do- \\
\hline 19. & Me Ap-19 & -do- \\
\hline 20. & Me Ap-20 & -do- \\
\hline 21. & Me Ap-21 & -do- \\
\hline 22. & Me Ap-22 & -do- \\
\hline 23. & $\mathrm{Me} \mathrm{Ap}-23$ & -do- \\
\hline 24. & Me Ap-24 & -do- \\
\hline 25. & Me Ap-25 & -do- \\
\hline
\end{tabular}


Int.J.Curr.Microbiol.App.Sci (2017) 6(9): 549-557

\begin{tabular}{|c|c|c|}
\hline 26. & Me Ap-26 & -do- \\
\hline 27. & Me Ap-34 & -do- \\
\hline 28. & Me Ap-35 & -do- \\
\hline 29. & Me Ap-36 & -do- \\
\hline 30. & Me Ap-37 & -do- \\
\hline 31. & Me Ap-38 & -do- \\
\hline 32. & Me Ap-39 & -do- \\
\hline 33. & Me Ap-40 & -do- \\
\hline 34. & Me Ap-41 & -do- \\
\hline 35. & Me Ap-42 & -do- \\
\hline 36. & Me Ap-43 & -do- \\
\hline 37. & Me Ap-44 & -do- \\
\hline 38. & Me Ap-45 & -do- \\
\hline 39. & Me Ap-46 & -do- \\
\hline 40. & Me Ap-47 & -do- \\
\hline 41. & Me Ap-48 & $\begin{array}{l}\text { AICRP on Tuber crops, Venkataramannagudem, Andhra } \\
\text { Pradesh }\end{array}$ \\
\hline 42. & Me Ap-49 & -do- \\
\hline 43. & Me Ap-50 & -do- \\
\hline 44. & Me Ap-51 & -do- \\
\hline 45. & Me Ap-52 & -do- \\
\hline 46. & Me Ap-53 & -do- \\
\hline 47. & Me Ap-54 & -do- \\
\hline 48. & Me Ap-55 & -do- \\
\hline 49. & Me Ap-56 & -do- \\
\hline 50. & Me Ap-57 & -do- \\
\hline 51. & Me Ap-58 & -do- \\
\hline 52. & Me Ap-59 & -do- \\
\hline 53. & Me Ap-60 & -do- \\
\hline 54. & Me Ap-61 & -do- \\
\hline 55. & Me Ap-62 & -do- \\
\hline 56. & Me Ap-63 & -do- \\
\hline 57. & Me Ap-64 & -do- \\
\hline 58. & Me Ap-65 & -do- \\
\hline 59. & Me Ap-66 & -do- \\
\hline 60. & Me Ap-67 & -do- \\
\hline 61. & Me Ap-68 & -do- \\
\hline 62. & Me Ap-69 & -do- \\
\hline 63. & Me Ap-70 & -do- \\
\hline 64. & Me Ap-71 & -do- \\
\hline 65. & Me Ap-72 & -do- \\
\hline 66. & Me Ap-73 & -do- \\
\hline 67. & Me Ap-74 & -do- \\
\hline 68. & Me Ap-75 & -do- \\
\hline 69. & Me Ap-76 & -do- \\
\hline 70. & Me Ap-77 & -do- \\
\hline 71. & Me Ap-78 & -do- \\
\hline 72. & Me Ap-79 & -do- \\
\hline 73. & Me Ap-29 & -do- \\
\hline 74. & Me Ap-30 & -do- \\
\hline 75. & Me Ap-31 & -do- \\
\hline 76. & Me Ap-32 & -do- \\
\hline 77. & Me Ap-33 & -do- \\
\hline 78. & H-165 (Check 1) & -do- \\
\hline 79. & H-226 (Check 2) & -do- \\
\hline 80. & Sree Vijaya (Check 3) & -do- \\
\hline
\end{tabular}


Table.2 Phenotypic and genotypic correlation coefficients for quantitative characters in cassava genotypes

\begin{tabular}{|c|c|c|c|c|c|c|c|c|c|c|c|c|c|c|c|c|}
\hline Character & & PL (cm) & $\begin{array}{l}\text { TLA } \\
(\mathbf{c m} 2)\end{array}$ & $\operatorname{PDM}(\%)$ & TDM (\%) & NLP & PH (cm) & STD (cm) & NSRT & NCRT & TL $(\mathbf{c m})$ & TD (cm) & HI (\%) & $\begin{array}{c}\text { STRCH } \\
(\%)\end{array}$ & $\begin{array}{l}\text { HCN } \\
(\mathbf{p p m})\end{array}$ & $\begin{array}{l}\text { PPD } \\
(\%)\end{array}$ \\
\hline \multirow{2}{*}{ PL (cm) } & rp & 1.000 & & & & & & & & & & & & & & \\
\hline & rg & 1.000 & & & & & & & & & & & & & & \\
\hline \multirow[t]{2}{*}{ TLA (cm2) } & rp & 0.043 & 1.000 & & & & & & & & & & & & & \\
\hline & rg & 0.044 & 1.000 & & & & & & & & & & & & & \\
\hline $\operatorname{PDM}(\%)$ & $\begin{array}{r}\mathbf{r n} \\
\mathbf{r g}\end{array}$ & $\begin{array}{l}-0.106 \\
-0.109\end{array}$ & $\frac{-0.011}{-0.015}$ & $\begin{array}{l}1.000 \\
1.000\end{array}$ & & & & & & & & & & & & \\
\hline \multirow{2}{*}{ TDM (\%) } & $\mathrm{ro}$ & 0.003 & 0.168 & $0.726^{* *}$ & 1.000 & & & & & & & & & & & \\
\hline & rg & 0.003 & 0.169 & $0.736^{* *}$ & 1.000 & & & & & & & & & & & \\
\hline \multirow{2}{*}{ NLP } & rp & -0.041 & $0.852^{* *}$ & 0.121 & $0.235^{*}$ & 1.000 & & & & & & & & & & \\
\hline & rg & -0.041 & $0.856^{* *}$ & 0.127 & $0.240^{*}$ & 1.000 & & & & & & & & & & \\
\hline \multirow{2}{*}{ PH (cm) } & rp & 0.102 & $0.337^{* *}$ & -0.021 & -0.120 & 0.187 & 1.000 & & & & & & & & & \\
\hline & rg & 0.102 & $0.344^{* *}$ & -0.016 & -0.117 & 0.188 & 1.000 & & & & & & & & & \\
\hline \multirow{2}{*}{ STD (cm) } & rp & $0.401^{* * *}$ & 0.136 & 0.103 & 0.105 & -0.037 & $0.448^{* *}$ & 1.000 & & & & & & & & \\
\hline & rg & $0.419^{* * *}$ & 0.135 & 0.094 & 0.109 & -0.038 & $0.474^{* *}$ & 1.000 & & & & & & & & \\
\hline \multirow{2}{*}{ NSRT } & rp & 0.103 & 0.186 & 0.007 & 0.031 & -0.006 & $0.278^{* *}$ & $0.228^{*}$ & 1.000 & & & & & & & \\
\hline & rg & 0.104 & 0.188 & 0.014 & 0.031 & -0.008 & $0.278^{* *}$ & $0.234^{*}$ & 1.000 & & & & & & & \\
\hline \multirow{2}{*}{ NCRT } & rp & -0.025 & $0.346^{* *}$ & 0.014 & 0.117 & $0.208^{*}$ & $0.304^{* *}$ & $0.287^{* *}$ & $0.800^{* *}$ & 1.000 & & & & & & \\
\hline & $\mathrm{rg}$ & -0.025 & $0.353^{* *}$ & 0.023 & 0.117 & $0.209^{*}$ & $0.306^{* *}$ & $0.339^{* *}$ & $0.816^{* *}$ & 1.000 & & & & & & \\
\hline \multirow{2}{*}{ TL (cm) } & rp & 0.027 & 0.129 & -0.036 & -0.075 & 0.093 & $0.315^{* *}$ & $0.296^{* *}$ & 0.184 & $0.219^{*}$ & 1.000 & & & & & \\
\hline & rg & 0.027 & 0.134 & -0.035 & -0.077 & 0.097 & $0.321^{* *}$ & $0.323^{* *}$ & 0.193 & $0.219^{*}$ & 1.000 & & & & & \\
\hline \multirow[b]{2}{*}{ TD $(\mathrm{cm})$} & rp & 0.053 & 0.089 & 0.109 & 0.112 & $0.209^{*}$ & $0.228^{*}$ & 0.117 & -0.011 & 0.070 & $0.364^{* *}$ & 1.000 & & & & \\
\hline & rg & 0.055 & 0.093 & 0.128 & 0.120 & $0.209^{*}$ & $0.227^{*}$ & 0.162 & -0.020 & 0.060 & $0.374^{* *}$ & 1.000 & & & & \\
\hline \multirow{2}{*}{ HI (\%) } & rp & 0.072 & 0.101 & $0.224^{*}$ & $0.203^{*}$ & 0.192 & 0.184 & 0.125 & -0.144 & -0.131 & 0.165 & $0.329^{* *}$ & 1.000 & & & \\
\hline & rg & 0.078 & 0.115 & $0.247^{*}$ & $0.218^{*}$ & $0.218^{*}$ & $0.221^{*}$ & 0.079 & -0.139 & -0.125 & 0.175 & $0.426^{* *}$ & 1.000 & & & \\
\hline \multirow{2}{*}{ STRCH (\%) } & rp & -0.188 & $0.227^{*}$ & -0.082 & -0.070 & $0.198^{*}$ & $0.313^{* *}$ & -0.173 & 0.148 & 0.132 & 0.178 & 0.084 & 0.118 & 1.000 & & \\
\hline & rg & -0.194 & $0.242^{*}$ & -0.075 & -0.081 & $0.206^{*}$ & $0.326^{* *}$ & -0.140 & 0.156 & 0.117 & 0.169 & 0.063 & 0.183 & 1.000 & & \\
\hline \multirow{2}{*}{ HCN (ppm) } & rp & -0.147 & $0.287^{* *}$ & -0.031 & -0.055 & $0.238^{*}$ & $0.228^{*}$ & 0.017 & $0.239^{*}$ & 0.113 & 0.001 & 0.008 & 0.155 & $0.294^{* *}$ & 1.000 & \\
\hline & rg & -0.149 & $0.290^{* *}$ & -0.029 & -0.050 & $0.230^{*}$ & $0.235^{*}$ & -0.005 & $0.240^{*}$ & 0.126 & 0.005 & 0.007 & 0.172 & $0.318^{* *}$ & 1.000 & \\
\hline \multirow{2}{*}{ PPD (\%) } & rp & -0.101 & 0.098 & -0.046 & 0.013 & $0.230^{*}$ & -0.128 & $-0.336^{* *}$ & -0.107 & 0.025 & -0.005 & -0.057 & -0.072 & 0.194 & -0.071 & 1.000 \\
\hline & rg & -0.101 & 0.098 & -0.047 & 0.012 & $0.230^{*}$ & -0.131 & $-0.358^{* *}$ & -0.108 & 0.026 & -0.005 & -0.055 & -0.081 & $0.204^{*}$ & -0.076 & 1.000 \\
\hline \multirow[b]{2}{*}{ TYLD/ha } & rp & 0.101 & $0.211^{*}$ & $0.208^{*}$ & $0.198^{*}$ & $0.207^{*}$ & $0.269^{* *}$ & $0.395^{* *}$ & $0.234^{*}$ & $0.322^{* *}$ & $0.420^{* *}$ & $0.623^{* *}$ & $0.300^{* *}$ & 0.014 & 0.031 & 0.021 \\
\hline & rg & 0.101 & $0.209^{*}$ & $0.214^{*}$ & $0.204^{*}$ & $0.206^{*}$ & $0.268^{* *}$ & $0.411^{* *}$ & $0.238^{*}$ & $0.327^{* *}$ & $0.423^{* *}$ & $0.641^{* *}$ & $0.315^{* *}$ & 0.020 & 0.024 & 0.019 \\
\hline
\end{tabular}

Note-" $5 \%$ level of significance, $1 \%$ level of significance

Where, PL-petiole length, TLA-total leaf area, PDM-plant dry matter content, TDM-tuber dry matter content, NLP- number of leaves per plant, PH-plant height,

STD-stem diameter, NSRT-no. of storage roots per plant, NCRT-No. of commercial roots per plant, TL-tuber length, TD-tuber diameter, HI-harvest index,

STRCH-starch content, HCN-hydrogen cyanide content, PPD-post harvest physiological deterioration, TYLD-tuber yield 
Table.3 Phenotypic and genotypic path coefficient analysis of quantitative characters in cassava genotypes

\begin{tabular}{|c|c|c|c|c|c|c|c|c|c|c|c|c|c|c|c|c|}
\hline Character & & PL (cm) & TLA (m2) & $\operatorname{PDM}(\%)$ & TDM (\%) & NLP & PH (cm) & $\operatorname{STD}(\mathrm{cm})$ & NSRT & NCRT & TL $(\mathbf{c m})$ & $\begin{array}{c}\text { TD } \\
(\mathbf{c m})\end{array}$ & HI (\%) & $\begin{array}{c}\%) \\
\text { STRCH }\end{array}$ & $\begin{array}{c}\text { HCN } \\
(\mathbf{p p m})\end{array}$ & $\begin{array}{l}\text { PPD } \\
(\%)\end{array}$ \\
\hline \multirow{2}{*}{ PL (cm) } & $\mathbf{R p}$ & -0.064 & -0.003 & 0.007 & 0.000 & 0.003 & -0.006 & -0.026 & -0.007 & 0.002 & -0.002 & -0.003 & -0.005 & 0.012 & 0.009 & 0.006 \\
\hline & $\mathbf{R g}$ & -0.109 & -0.005 & 0.012 & 0.000 & 0.004 & -0.011 & -0.046 & -0.011 & 0.003 & -0.003 & -0.006 & -0.008 & 0.021 & 0.016 & 0.011 \\
\hline \multirow{2}{*}{ TLA (m2) } & $\mathbf{R p}$ & -0.001 & 0.028 & 0.000 & -0.005 & -0.024 & -0.009 & -0.004 & -0.005 & -0.010 & -0.004 & -0.002 & -0.003 & -0.006 & $\begin{array}{r}-0.008 \\
\end{array}$ & -0.003 \\
\hline & Rg & -0.001 & 0.015 & 0.000 & -0.003 & -0.013 & -0.005 & -0.002 & -0.003 & -0.005 & -0.002 & -0.001 & -0.002 & -0.004 & $\begin{array}{r}-0.004 \\
\end{array}$ & -0.001 \\
\hline \multirow{2}{*}{ PDM (\%) } & $\mathbf{R n}$ & -0.010 & -0.001 & 0.090 & 0.066 & 0.011 & -0.002 & 0.009 & 0.001 & 0.001 & -0.003 & 0.010 & 0.020 & -0.007 & $-0,003$ & -0.004 \\
\hline & Rg & -0.008 & -0.001 & 0.072 & 0.053 & 0.009 & -0.001 & 0.007 & 0.001 & 0.002 & -0.003 & 0.009 & 0.018 & -0.005 & -0.002 & -0.003 \\
\hline TDM (\%) & $\begin{array}{l}\mathbf{R n} \\
\mathbf{R g}\end{array}$ & $\begin{array}{l}0.000 \\
0.000\end{array}$ & $\begin{array}{l}-0.003 \\
-0.003\end{array}$ & $\begin{array}{l}-0.013 \\
-0.011\end{array}$ & $\begin{array}{l}-0.018 \\
-0.015\end{array}$ & $\begin{array}{l}-0.004 \\
-0.004\end{array}$ & 0.002 & $\begin{array}{l}-0.002 \\
-0.002\end{array}$ & $\frac{-0.001}{0.000}$ & $\begin{array}{r}-0.002 \\
-0.002\end{array}$ & $\begin{array}{l}0.001 \\
0.001\end{array}$ & $\begin{array}{l}-0.002 \\
-0.002\end{array}$ & $\begin{array}{l}-0.004 \\
-0.003\end{array}$ & 0.001 & $\begin{array}{l}0.001 \\
0.001\end{array}$ & $\begin{array}{l}0.000 \\
0.000\end{array}$ \\
\hline \multirow{2}{*}{ NLP } & $\mathbf{R p}$ & -0.003 & 0.059 & 0.008 & 0.016 & 0.069 & 0.013 & -0.003 & 0.000 & 0.014 & 0.006 & 0.014 & 0.013 & 0.014 & 0.016 & 0.016 \\
\hline & $\mathbf{R g}$ & -0.003 & 0.073 & 0.011 & 0.020 & 0.085 & 0.016 & -0.003 & -0.001 & 0.018 & 0.008 & 0.018 & 0.019 & 0.017 & 0.020 & 0.020 \\
\hline \multirow{2}{*}{ PH (cm) } & $\mathbf{R p}$ & -0.008 & -0.026 & 0.002 & 0.009 & -0.014 & -0.076 & -0.034 & -0.021 & -0.023 & -0.024 & -0.017 & -0.014 & -0.024 & -0.017 & 0.010 \\
\hline & rg & -0.010 & -0.034 & 0.002 & 0.012 & -0.019 & -0.100 & -0.048 & -0.028 & -0.031 & -0.032 & -0.023 & -0.022 & -0.033 & -0.024 & 0.013 \\
\hline \multirow{2}{*}{ STD (cm) } & rp & 0.136 & 0.046 & 0.035 & 0.035 & -0.012 & 0.152 & 0.339 & 0.077 & 0.097 & 0.100 & 0.040 & 0.042 & -0.059 & 0.006 & -0.114 \\
\hline & rg & 0.168 & 0.054 & 0.038 & 0.044 & -0.015 & 0.190 & 0.401 & 0.094 & 0.136 & 0.130 & 0.065 & 0.032 & -0.056 & -0.002 & -0.144 \\
\hline \multirow{2}{*}{ NSRT } & $\mathbf{r p}$ & 0.018 & 0.032 & 0.001 & 0.005 & -0.001 & 0.048 & 0.039 & 0.171 & 0.137 & 0.031 & -0.002 & -0.025 & 0.025 & 0.041 & -0.018 \\
\hline & rg & 0.028 & 0.051 & 0.004 & 0.008 & -0.002 & 0.076 & 0.064 & 0.272 & 0.222 & 0.053 & -0.006 & -0.038 & 0.043 & 0.065 & -0.029 \\
\hline \multirow{2}{*}{ NCRT } & rp & -0.002 & 0.023 & 0.001 & 0.008 & 0.014 & 0.020 & 0.019 & 0.054 & 0.067 & 0.015 & 0.005 & -0.009 & 0.009 & 0.008 & 0.002 \\
\hline & $\mathrm{rg}$ & 0.001 & -0.016 & -0.001 & -0.005 & -0.010 & -0.014 & -0.015 & -0.037 & -0.046 & -0.010 & -0.003 & 0.006 & -0.005 & -0.006 & -0.001 \\
\hline \multirow{2}{*}{ TL (cm) } & rp & 0.003 & 0.013 & -0.004 & -0.007 & 0.009 & 0.032 & 0.030 & 0.018 & 0.022 & 0.100 & 0.036 & 0.017 & 0.018 & 0.000 & -0.001 \\
\hline & $\mathrm{rg}$ & 0.002 & 0.010 & -0.003 & -0.006 & 0.007 & 0.024 & 0.024 & 0.014 & 0.016 & 0.074 & 0.028 & 0.013 & 0.013 & 0.000 & 0.000 \\
\hline \multirow[b]{2}{*}{ TD $(\mathbf{c m})$} & rp & 0.028 & 0.046 & 0.057 & 0.058 & 0.109 & 0.119 & 0.061 & -0.006 & 0.037 & 0.190 & 0.521 & 0.171 & 0.044 & 0.004 & -0.030 \\
\hline & $\mathrm{rg}$ & 0.029 & 0.049 & 0.068 & 0.063 & 0.111 & 0.120 & 0.086 & -0.011 & 0.032 & 0.198 & 0.530 & 0.226 & 0.033 & 0.004 & -0.029 \\
\hline \multirow{2}{*}{ HI (\%) } & rp & 0.008 & 0.012 & 0.026 & 0.024 & 0.022 & 0.022 & 0.015 & -0.017 & -0.015 & 0.019 & 0.038 & 0.117 & 0.014 & 0.018 & -0.008 \\
\hline & rg & 0.008 & 0.012 & 0.026 & 0.023 & 0.023 & 0.024 & 0.008 & -0.015 & -0.013 & 0.019 & 0.046 & 0.107 & 0.020 & 0.018 & -0.009 \\
\hline \multirow{2}{*}{ STRCH (\%) } & $\mathbf{r p}$ & 0.011 & -0.013 & 0.005 & 0.004 & -0.011 & -0.018 & 0.010 & -0.008 & -0.007 & -0.010 & -0.005 & -0.007 & -0.056 & -0.016 & -0.011 \\
\hline & $\mathrm{rg}$ & 0.011 & -0.014 & 0.004 & 0.005 & -0.012 & -0.018 & 0.008 & -0.009 & -0.007 & -0.010 & -0.004 & -0.010 & -0.056 & -0.018 & -0.012 \\
\hline \multirow{2}{*}{ HCN (ppm) } & rp & 0.002 & -0.004 & 0.000 & 0.001 & -0.004 & -0.003 & 0.000 & -0.004 & -0.002 & 0.000 & 0.000 & -0.002 & -0.004 & -0.015 & 0.001 \\
\hline & $\mathrm{rg}$ & 0.004 & -0.008 & 0.001 & 0.001 & -0.007 & -0.007 & 0.000 & $\begin{array}{l}-0.007 \\
\end{array}$ & -0.004 & 0.000 & 0.000 & -0.005 & -0.009 & -0.029 & 0.002 \\
\hline \multirow{2}{*}{ PPD (\%) } & rp & -0.018 & 0.017 & -0.008 & 0.002 & 0.040 & -0.022 & -0.059 & -0.019 & 0.004 & -0.001 & -0.010 & -0.013 & 0.034 & -0.012 & 0.175 \\
\hline & rg & -0.021 & 0.020 & -0.010 & 0.002 & 0.047 & -0.027 & -0.072 & -0.022 & 0.005 & -0.001 & -0.011 & -0.016 & 0.041 & -0.015 & 0.202 \\
\hline \multirow[b]{2}{*}{ TYLD/ha } & $\mathbf{r p}$ & 0.101 & 0.211 & 0.208 & 0.198 & 0.207 & 0.269 & 0.395 & 0.234 & 0.322 & 0.420 & 0.623 & 0.300 & 0.014 & 0.031 & 0.021 \\
\hline & rg & 0.101 & 0.209 & 0.214 & 0.204 & 0.206 & 0.268 & 0.411 & 0.238 & 0.327 & 0.423 & 0.641 & 0.315 & 0.020 & 0.024 & 0.019 \\
\hline
\end{tabular}

Phenotypic Residual effect $=0.6238$; Genotypic Residual effect $=0.6346$; Diagonal (under lined) values indicate direct effects

Where, PL-petiole length, TLA-total leaf area, PDM-plant dry matter content, TDM-tuber dry matter content, NLP- number of leaves per plant, PH-plant height,

STD-stem diameter, NSRT-number of storage roots per plant, NCRT-number of commercial roots per plant, TL-tuber length, TD-tuber diameter, HI-harvest

index, STRCH-starch content, HCN-hydrogen cyanide content, PPD-post harvest physiological deterioration, TYLD-tuber yield 
The path coefficient analysis which splits total correlation coefficient of different characters into direct and indirect effects on tuber yield in such a manner that the sum of direct and indirect effects is equal to total genotypic correlation as presented in table 3 and figure 2 . The path analysis indicated that stem diameter $(0.339 \mathrm{P}, 0.401 \mathrm{G})$, number of storage roots per plant $(0.171 \mathrm{P}, 0.272 \mathrm{G})$, tuber length $(0.100 \mathrm{P}, 0.074 \mathrm{G})$, tuber diameter content $(0.521 \mathrm{P}, 0.530 \mathrm{G})$, harvest index $(0.117 \mathrm{P}, 107 \mathrm{G})$ and post-harvest physiological deterioration $(0.175 \mathrm{P}, 0.202 \mathrm{G})$ had direct positive effects on tuber yield at both phenotypic and genotypic levels. The characters like total leaf area $(0.028 \mathrm{P}, 0.015$ $\mathrm{G})$, plant dry matter content (0.090 P, 0.072 $\mathrm{G})$, number of leaves per plant (0.069 P, $0.085 \mathrm{G})$ and number of commercial roots per plant $(0.067 \mathrm{P})$ also had positive direct effect on tuber yield per hectare. The results are in accordance with Babu Rao et al., (2015) for stem diameter and Adeniji et al., (2011) and Babu Rao et al., (2015) for plant dry matter content, number of storage roots per plant and Mulaulem and Ayenew (2012) and Babu Rao et al., (2015) for tuber dry matter content. Rekha et al., (1991) and Babu Rao et al., (2015) were also reported similar results for number of leaves per plant. Similarly Rajendran et al., (1985), Mulaulem and Ayenew (2012) and Babu Rao et al., (2015) observed high positive direct effect on tuber yield with respect to tuber diameter.

The negative direct effects on tuber yield was recorded for petiole length $(-0.064 \mathrm{P},-0.109$ $\mathrm{G})$, tuber dry matter content $(-0.018 \mathrm{P},-0.015$ $\mathrm{G})$, plant height $(-0.076 \mathrm{P},-0.100 \mathrm{G})$, starch content (-0.056 P, -0.056 G), HCN content ($0.015 \mathrm{P},-0.029 \mathrm{G})$ at both phenotypic and genotypic levels. These results for tuber dry matter content and plant height are in accordance with the earlier reports by Mulaulem and Ayenew (2012) and Babu Rao et al., (2015). Negative direct effect of $\mathrm{HCN}$ content on tuber yield was in conformity with the results of Babu Rao et al., (2015). Path analysis revealed that major emphasis should be laid on selection process with more stem diameter, number of storage roots per plant, tuber length, tuber diameter content, harvest index and post-harvest physiological deterioration and there should be economic balance among these traits to get higher tuber yield.

\section{References}

Adeniji, O.T., Odo, P.E. and Ibrahim, B. 2011. Genetic relationships and selection indices for cassava root yield in Adamawa State, Nigeria. African Journal of Agricultural Research. 6(13): 2931-2934.

Al-Jibouri, H.A., P.A. Miller, and H.V. Robinson. 1958. Genotypic and environmental variances and covariances in an upland cotton cross of interspecific origin. Agron. J. 50:533536.

Alves, A.A.C., 2002. Cassava Botany and Physiology. In: R.J.Hillocks, JM Thresh, A Bellotti, Eds., Cassava: Biology, Production and Utilization: 6790.

Anonymous, 2014. http://www.nhb.gov.in/ area production.html.

Babu Rao, B., Ashok, P., Ramanandam, G. and Sasikala, K. 2015. Trait association and path coefficient analyses in cassava, International Journal of Vegetable Science. 21(4): 402-415.

Danquah, J. A., Gracen, V. E., Offei, S. K., Asante, I. K. and Aduening, J. M. 2016. Agronomic performance and genotypic diversity for morphological traits among cassava genotypes in the Guinea Savannah ecology of Ghana. Journal of crop Science and Biotechnology.19 (1): 99-108.

Dewey, O.R., and K.H. Lu. 1959. A 
correlation and path analysis of components of crested wheat grass seed production. Agron. J. 51:515-518.

Fisher, R.A., and F. Yates. 1963. Statistical tables for biological, agricultural and medical research. Oliver and Boyd, London.

Goulden, C.H. 1959. Methods of statistical analysis. John Wiley \& Sons, New York.

Johnson, H.W., Robinson, H.F. and Comstock, R.E. 1955. Estimates of genetic and environmental variability in soybean. Agronomy Journal. 47: 314318.

Kang, M.S., 1994. Applied quantitative genetics. Dept. Agronomy, Louisiana State Univ., Baton Rouge, La.

Kawano, K., and W.M. Fukuda. 1998. Yield improvement in multistage breeding program for cassava. Crop Sci. 38:325334.

Kundy, A.C., Mkamilo, G.S. and Misangu, R.N. 2015. Genetic variability among six traits in twelve cassava (Manihot esculenta Crantz) genotypes in Southern Tanzania. Journal of Natural Sciences Research. 5(12): 33-38.
Mulualem, T., and Ayenew, B. 2012. Correlation and path coefficient analysis of cassava (Manihot esculenta Crantz) at Jimma, Southwestern, Ethiopia. Journal of Natural Sciences Research. 2(9): 1-7.

Nageswari, K., and Palaniswamy, V. 2011. Correlation and Genetic variability studies in cassava (Manihot esculenta Crantz). NSCFT, CTCRI proceedings. $219-222$.

Rajendran, P.G., Lakshmi, K.R. and Unnikrishnan, M. 1985. Genetic and path coefficient analysis in cassava. National Symposium on Tropical tuber crops. 1-5.

Rekha, V.R., Manikantan, N.P., Sreekumar, S.G., Balakrishnan, A.R. and Pillai, M.R.C. 1991. Path analysis of yield components in a few cassava cultivars. Journal of Root Crops. 17(1): 35-38.

Sreekumari, M.T., and Abraham, K. 1991. Correlation studies in shade grown cassava. Journal of Root Crops. 17 (1): 56-59.

Wright, S., 1921. Correlation and causation. J. Agr. Res. 20:557-585.

\section{How to cite this article:}

Babu Rao, B., D.V. Swami, P. Ashok, B. Kalyana Babu, D. Ramajayam and Sasikala, K. 2017. Correlation and Path Coefficient Analysis of Cassava (Manihot esculenta Crantz) Genotypes. Int.J.Curr.Microbiol.App.Sci. 6(9): 549-557. doi: https://doi.org/10.20546/ijcmas.2017.609.066 